\title{
Holzerntepotenzial im Schweizer Wald: Simulation von Bewirtschaftungsszenarien
}

Golo Stadelmann Anne Herold Markus Didion Beatriz Vidondo Andres Gomez Esther Thürig
Eidgenössische Forschungsanstalt für Wald, Schnee und Landschaft $(\mathrm{CH})$ * Eidgenössische Forschungsanstalt für Wald, Schnee und Landschaft $(\mathrm{CH})$ Eidgenössische Forschungsanstalt für Wald, Schnee und Landschaft $(\mathrm{CH})$ Eidgenössische Forschungsanstalt für Wald, Schnee und Landschaft $(\mathrm{CH})$ Eidgenössische Forschungsanstalt für Wald, Schnee und Landschaft $(\mathrm{CH})$ Eidgenössische Forschungsanstalt für Wald, Schnee und Landschaft $(\mathrm{CH})$

\begin{abstract}
Holzerntepotenzial im Schweizer Wald: Simulation von Bewirtschaftungsszenarien
In den letzten zehn Jahren ist der Holzvorrat in der Schweiz um 3\% gestiegen, stärker war die Vorratszunahme im Alpenraum, während der Hektarvorrat im Mittelland gesunken ist. Mit dem Waldentwicklungsmodell Massimo simulierten wir fünf Bewirtschaftungsszenarien über einen Zeitraum von 100 Jahren. Im Basisszenario «konstanter Vorrat» können im Mittel jährlich 7.7 Mio. $\mathrm{m}^{3}$ Derbholz geerntet werden. Tiefer ist das Erntepotenzial im Szenario «Vorratsanstieg» (6.3 Mio. $\left.\mathrm{m}^{3}\right)$. Höhere Erntepotenziale ergeben sich in den Szenarien «kontinuierlich hoher Zuwachs» (7.8 Mio. $\left.\mathrm{m}^{3}\right)$, «grosse Nadelholznachfrage» (8.5 Mio. $\left.\mathrm{m}^{3}\right)$ und «grosse Energie- und Chemieholznachfrage» (8.1 Mio. $\mathrm{m}^{3}$ ). Bei allen Szenarien steigen die Holzerntekosten je Kubikmeter über die Zeit an, wobei dieser Anstieg für das Basisszenario am geringsten ausfällt. Das Weiterführen der bisherigen Bewirtschaftung (Szenario Vorratsanstieg) dürfte aufgrund zunehmender Bestandesdichte und zunehmenden Vorrats zu einem höheren Risiko für Störungen (Wind, Schneebruch, Borkenkäfer) führen, während die regionale Übernutzung im Mittelland die Holzverfügbarkeit einschränkt. Im Alpenraum könnte eine Stabilisierung des Vorrats (Szenario konstanter Vorrat) die Resistenz des Waldes verbessern, ist jedoch mit hohen Erntekosten je Kubikmeter verbunden. Die Bewirtschaftungsszenarien sollen Entscheidungsträgern eine Hilfe bieten, um geeignete Strategien zur Vorrats- und Nutzungsplanung festzulegen und deren Kosten abzuschätzen.
\end{abstract}

Keywords: timber-harvesting potential, Massimo, forest management, empirical simulation model, management scenario doi: $10.3188 /$ szf. 2016.0152

*Zürcherstrasse 111, CH-8903 Birmensdorf, E-Mail golo.stadelmann@wsl.ch
$\mathrm{D}$ er Holzvorrat nimmt in der Schweiz seit dem ersten Landesforstinventar (LFI1; 1983/ 1985) stetig zu. Ohne Berücksichtigung der ebenfalls zunehmenden Waldfläche ist der Holzvorrat in der Schweiz seit 1995 um 3\% gestiegen und beträgt heute auf der zugänglichen Waldfläche des LFI4b (2009/2013) $350 \mathrm{~m}^{3} / \mathrm{ha}$. Werden die Vorratsveränderungen jedoch nach Produktionsregion aufgeschlüsselt, differenziert sich diese Entwicklung sehr: Im Mittelland sank der Vorrat um 11\%, während in den Alpen $(+14 \%)$ und vor allem auf der Alpensüdseite (+30\%) eine starke Zunahme des Vorrats beobachtet wurde (Camin et al 2015). Diese Vorratsentwicklung ist auf regionale Über- respektive Unternutzungen zurückzuführen: Im Mittelland wurden zwischen 1995 und 2013 123\% des Nettozuwachses genutzt, auf der Alpensüdseite hingegen nur 20\% (Camin \& Fischer 2015). Wenn die Nutzung während eines kurzen Zeitraums über dem Zuwachs liegt, dürfte das die langfristigen Holzerntepotenzi- ale, d.h. die langfristige Verfügbarkeit von Nutzholz, nicht gefährden. Auch ein kurzfristiger Nutzungsverzicht gefährdet die Holzerntepotenziale nicht, da diese dadurch tendenziell steigen. Langfristig kann sich jedoch starke Unter- oder Übernutzung negativ auf die Produktivität des langsam reagierenden Waldsystems auswirken.

Um die langfristigen Auswirkungen spezifischer Bewirtschaftungsstrategien abzuschätzen, werden oft räumlich repräsentative Prognosemodelle wie «Weham» (Bösch 2004), «Prognaus» (Monserud \& Sterba 1996) oder «EFISCEN» (Nabuurs et al 2000) eingesetzt. Diese Modelle wurden entwickelt, um die Entwicklung forstlicher Ressourcen und deren Nutzung auf der Basis von Stichprobeninventuren und Bewirtschaftungsszenarien zu simulieren. In der Schweiz wurde dazu das Modell Massimo (Kaufmann 2001) entwickelt. Mit diesem Modell wurde die erste nationale Studie zum Holzerntepotenzial erarbeitet (Hofer et al 2011, Kaufmann 2011), es wurden die 
Möglichkeiten zur Kohlenstoffspeicherung im Wald untersucht (Thürig \& Kaufmann 2008, Werner et al 2010) und zur Anrechnung der Waldsenken und -quellen in der zweiten Verpflichtungsperiode des Kyoto-Protokolls wurde ein Referenzwert modelliert, der sogenannte Forest Management Reference Level (FMRL), beschrieben in FOEN (2015). Massimo wird zudem verwendet, um Energieholzpotenziale (Thees et al 2013) und regionale Bewirtschaftungsszenarien zu untersuchen (Stadelmann et al 2015).

Mit den Ergebnissen des LFI4b konnten neue Bewirtschaftungsszenarien zum Holzerntepotenzial im Schweizer Wald definiert werden. Die Szenarien berücksichtigen einerseits die neusten Inventurdaten und behandeln andererseits aktuelle waldpolitische Themen. Konkret wird in dieser Studie das Holzerntepotenzial des Schweizer Waldes simuliert, um folgende drei Fragen $\mathrm{zu}$ beantworten: 1) Welche Holznutzung ist möglich, wenn der Vorrat in allen Wirtschaftsregionen stabil gehalten wird? 2) Wie entwickelt sich der Wald, wenn die Nutzung in allen Wirtschaftsregionen auf dem Niveau zwischen LFI3 (2004/2006) und LFI4b konstant gehalten wird? 3) Können die langfristigen Nutzungsmengen und Zuwächse durch eine Vorratssenkung gesteigert werden? Die Ergebnisse dieser Studie zeigen ein breites Spektrum der Waldentwicklung und weisen auf potenzielle Auswirkungen verschiedener Bewirtschaftungsstrategien hin. Damit bildet die Studie eine Grundlage zur Diskussion langfristiger nationaler Bewirtschaftungsziele. Die Simulationsergebnisse aus Massimo stellen das Holzerntepotenzial ohne Berücksichtigung sozioökologischer oder ökonomischer Nutzungsfaktoren dar. In der Studie von Taverna et al (2016, dieses Heft) werden solche Faktoren berücksichtigt, um aus dem Holzerntepotenzial dieser Studie das nachhaltig verfügbare Holznutzungspotenzial abzuleiten.

\section{Material und Methoden}

\section{Das Modell Massimo}

In Massimo (Management Scenario Simulation Model) werden die Auswirkungen verschiedener Bewirtschaftungsszenarien auf die Bestandesentwicklung und die Verfügbarkeit forstlicher Ressourcen simuliert (Kaufmann 2001, 2011). Für jeden 10-Jahres-Schritt werden Informationen zum Bestandesaufbau und zur Holzernte generiert. Der Simulationszeitraum beträgt maximal 100 Jahre, da die Unsicherheiten des Modells mit jedem simulierten Zeitschritt zunehmen.

Massimo besteht im Wesentlichen aus den Prozessen Wachstum, Bewirtschaftung und Mortalität, Zuwachsreaktion nach Durchforstungen, Einwuchs und Verjüngung. Der einzelbaumweise Zuwachs wird für jede Baumart mit einer Wachstumsfunk- tion berechnet, die den Brusthöhendurchmesser, Standortfaktoren (Produktionsregion, Gesamtwuchsleistung, Höhe über Meer) und Bestandesfaktoren (Grundfläche, Bestandesalter, Konkurrenz, Schichtzugehörigkeit) berücksichtigt. Diese Wachstumsfunktionen werden zudem verwendet, um Zuwachsreaktionen nach Durchforstungen (vgl. Thürig et al 2005) und den Einwuchs über die Kluppschwelle $(12 \mathrm{~cm}) \mathrm{zu}$ modellieren.

Im Bewirtschaftungs- und Mortalitätsmodul wird jedem abgegangenen Baum eine Ursache zugeordnet: Sturmschaden, andere natürliche Mortalität, Durchforstung oder Schirmhieb. Zusätzlich zu Sturmschaden und anderen Zwangsnutzungen wird in wenig durchforsteten Beständen eine dichteabhängige Mortalität simuliert, die aus Ertragskundedaten hergeleitet wurde. Das Ausmass der natürlichen Mortalität hängt vom Bewirtschaftungsszenario ab und steigt mit zunehmender Bestandesdichte. Durchforstungen sind als Auslesedurchforstung implementiert, wobei die Durchforstungsstärke im gleichförmigen Hochwald 30\% und im ungleichförmigen Hochwald 25\% der Grundfläche beträgt. Das Durchforstungsintervall ist abhängig vom Grundflächenzuwachs, weshalb in Massimo wüchsige Bestände an Tieflagen öfter durchforstet werden als solche an Hochlagen. Die Anzahl der zu verjüngenden Bestände ergibt sich aus dem Bestandesalter und der Umtriebszeit, die von der Standortgüte abhängt (Kaufmann 2001). Die Verjüngung wird mit einem Schirmhieb eingeleitet, wobei 80\% der Oberschichtbäume entnommen werden. Die restlichen Bäume werden in der kollinen, der submontanen und der unteren montanen Stufe nach 20 Jahren und in den übrigen Stufen nach 30 Jahren entnommen. Die Verjüngung von Wald, der vor Steinschlag, Lawinen oder Hangmuren schützt, erfolgt mittels Durchforstungen: Alle 20 Jahre werden $40 \%$ der zu Verjüngungsbeginn vorhandenen Oberschichtsbäume entnommen. Das Erreichen der Vorrats- oder Nutzungsziele eines Bewirtschaftungsszenarios wird gesteuert, indem die Durchforstungsintervalle und Umtriebszeiten variiert werden.

Unabhängig vom Szenario ist in Massimo eine minimale Pflege des Schutzwalds und des ungleichförmigen Hochwalds gewährleistet, weil diese auch gepflegt und durchforstet werden, wenn die Definition des Szenarios die Nutzung stark einschränkt.

Auf Probeflächen, die verjüngt werden, wird Anwuchs generiert. Die Anzahl neuer Pflanzen im Jungwuchs und deren Mischung basiert auf einer zufällig gezogenen Jungwaldstichprobe mit vergleichbaren Standort- und Wuchsbedingungen. Zusätzlich kann bei der Definition eines Bewirtschaftungsszenarios die Baumartenmischung der Verjüngung beeinflusst werden. Die von der Waldgesellschaft abhängigen Definitionen des Nadelholzanteils «optimal», «empfohlen» und «maximal zulässig», abge- 
leitet aus der Wegleitung «Nachhaltigkeit und Erfolgskontrolle im Schutzwald» (NaiS; Frehner et al 2005), können im Modell gewählt werden.

Zur Herleitung der Holzerntekosten wurde das Holzernte-Produktivitätsmodell $\mathrm{HeProMo}^{1}$ in Massimo integriert, sodass die Kosten für die geernteten Mengen für jede LFI-Probefläche und für jeden Simulationsdurchgang einzeln berechnet werden können. Unter der Annahme, dass ein Eingriff stattfindet, wenn er innerhalb gewisser Kosten durchführbar ist, wurden die Holzerntekosten zu drei Klassen aggregiert: <80 CHF/m³ (durchführbar), 80-150 CHF/ $\mathrm{m}^{3}$ (durchführbar mit Beiträgen), >150 CHF/m³ (Eingriff wenig wahrscheinlich).

\section{Definition der Bewirtschaftungsszenarien}

Die Simulation von Bewirtschaftungsszenarien ermöglicht es, die Auswirkungen einer bestimmten Bewirtschaftungsweise auf den Schweizer Wald abzuschätzen. Gemeinsam mit einer vom Bundesamt für Umwelt (BAFU) koordinierten Expertengruppe wurden fünf Bewirtschaftungsszenarien für den Schweizer Wald erarbeitet (Tabelle 1), die aktuelle Probleme und neue Herausforderungen der Waldbewirtschaftung behandeln.

Das Basisszenario (A) hat zum Ziel, den Vorrat gemäss LFI4b in allen Wirtschaftsregionen konstant zu halten. Die Summe aller Abgänge (Nutzung und Mortalität) entspricht somit dem Bruttozuwachs, wodurch sowohl Über- als auch Unternutzung ausgeschlossen werden.

Unter Szenario Vorratsanstieg (B) wird die Nutzung in allen Wirtschaftsregionen konstant gehal- ten auf dem Niveau zwischen LFI3 und LFI4b. Dieses Szenario dürfte die mittelfristigen Auswirkungen der aktuellen Bewirtschaftung am besten aufzeigen.

Das Szenario kontinuierlich hoher Zuwachs (C) hat das Ziel, den langfristigen Zuwachs durch eine Vorratsabsenkung zu steigern und dabei die kurzbis mittelfristigen Zuwachsverluste klein zu halten.

Das Szenario grosse Nachfrage nach Nadelrundholz (D) hat zum Ziel, eine steigende Nachfrage nach Nadelrundholz zu decken. Kurz- bis mittelfristig kann dies mittels Vorratsabsenkung umgesetzt werden. Um die Produktion von Nadelrundholz langfristig zu steigern, wird der Nadelholzanteil in der Verjüngung erhöht.

Das Szenario grosse Nachfrage nach Energie- und Chemieholz (E) soll die Holzproduktion maximieren, wobei die Zieldurchmesser keine Rolle spielen. Durch eine rasche Vorratsabsenkung und eine Verkürzung der Umtriebszeit werden Zuwachs und Nutzung gesteigert. Als Ausgleich für die intensive Bewirtschaftung werden Waldreservate ausgeschieden, einerseits an seltenen Waldstandorten und andererseits an Orten, wo sich die Nutzung wenig lohnt und daher schon seit längerer Zeit keine Bewirtschaftung mehr stattgefunden hat.

Alle Ergebnisse beziehen sich auf den gemeinsam zugänglichen Wald ohne Gebüschwald der Inventuren LFI2 (1993/1995) und LFI3 (2004/

\footnotetext{
1 FRUTIG F, HOLM S, LEMM R, PEDOLIN D, THEES O (2015) Kalkulation von Holzerntearbeiten. Das Produktivitätsmodell HeProMo. Version 2015. Download von www.waldwissen.net/ technik/holzernte/kalkulation/wsl_hepromo/index_DE (15.2.2016)
}

\begin{tabular}{|c|c|c|}
\hline Szenario & Vorrats- respektive Nutzungsziele & Weitere Vorgaben \\
\hline A: Basisszenario & $\begin{array}{l}\text { Konstanter Vorrat auf dem Niveau von } \\
\text { LFI4b (2009/2013) in allen Wirtschafts- } \\
\text { regionen }\end{array}$ & $\begin{array}{l}\text { Mortalität: } 15 \% \text { der Abgänge } \\
\text { Verjüngung: empfohlene Nadelholzanteile } \\
\text { Sturm: ja, Periodizität } 15 \text { Jahre } \\
\text { Reservate: keine }\end{array}$ \\
\hline B: Vorratsanstieg & $\begin{array}{l}\text { Konstante Nutzung wie zwischen LFI3 } \\
(2004 / 2006) \text { und LFI4b (2009/2013) in } \\
\text { allen Wirtschaftsregionen }\end{array}$ & $\begin{array}{l}\text { Mortalität: } 15 \% \text { der Abgänge } \\
\text { Verjüngung: empfohlene Nadelholzanteile } \\
\text { Sturm: ja, Periodizität } 15 \text { Jahre } \\
\text { Reservate: keine }\end{array}$ \\
\hline $\begin{array}{l}\text { C: kontinuierlich hoher } \\
\text { Zuwachs }\end{array}$ & $\begin{array}{l}\text { Vorratsabsenkung auf } 300 \mathrm{~m}^{3} / \text { ha bis } 2046 \text {, } \\
\text { danach konstanter Vorrat }\end{array}$ & $\begin{array}{l}\text { Mortalität: } 15 \% \text { der Abgänge } \\
\text { Verjüngung: empfohlene Nadelholzanteile } \\
\text { Sturm: ja, Periodizität } 15 \text { Jahre } \\
\text { Reservate: keine }\end{array}$ \\
\hline $\begin{array}{l}\text { D: grosse Nachfrage nach } \\
\text { Nadelrundholz }\end{array}$ & $\begin{array}{l}\text { Vorratsabsenkung auf } 250 \mathrm{~m}^{3} / \text { ha bis } \\
2046 \text {, danach Anstieg des Vorrats auf } \\
300-330 \mathrm{~m}^{3} / \text { ha }\end{array}$ & $\begin{array}{l}\text { Mortalität: } 10 \% \text { der Abgänge } \\
\text { Verjüngung: maximal zulässige Nadelholzanteile } \\
\text { Sturm: ja, Periodizität } 15 \text { Jahre } \\
\text { Reservate: keine }\end{array}$ \\
\hline $\begin{array}{l}\text { E: grosse Nachfrage nach } \\
\text { Energie- und Chemieholz }\end{array}$ & $\begin{array}{l}\text { Schweizweite Vorratsabsenkung bis } \\
2046 \text { auf } 250 \mathrm{~m}^{3} / \text { ha mit regional unter- } \\
\text { schiedlichen Vorratszielen (Mittelland: } \\
200 \mathrm{~m}^{3} / \mathrm{ha} \text {; Jura, Voralpen, Wallis, Alpen- } \\
\text { südseite: } 250 \mathrm{~m}^{3} / \mathrm{ha} \text {; Alpen ohne Wallis: } \\
300 \mathrm{~m}^{3} / \mathrm{ha} \text { ), danach konstanter Vorrat in } \\
\text { allen Regionen }\end{array}$ & $\begin{array}{l}\text { Mortalität: } 10 \% \text { der Abgänge } \\
\text { Verjüngung: empfohlene Nadelholzanteile } \\
\text { Sturm: ja, Periodizität } 15 \text { Jahre } \\
\text { Reservate: seltene Waldgesellschaften oder } \\
\text { keine Nutzung seit längerer Zeit (Jura > } 55 \text { J, } \\
\text { Mittelland }>25 \text { J, Voralpen >110 J, Alpen >120 J, } \\
\text { Alpensüdseite }>150 \text { J) }\end{array}$ \\
\hline
\end{tabular}

Tab 1 Definition der Bewirtschaftungsszenarien. allen Regionen 


\begin{tabular}{|c|c|c|c|c|c|c|}
\hline \multirow[t]{2}{*}{ Merkmal } & \multirow[t]{2}{*}{ Zeitraum } & \multicolumn{5}{|c|}{ Szenario } \\
\hline & & $\begin{array}{c}\text { A: Basisszenario } \\
\text { (konstanter } \\
\text { Vorrat) }\end{array}$ & $\begin{array}{l}\text { B: Vorrats- } \\
\text { anstieg }\end{array}$ & $\begin{array}{l}\text { C: kontinuierlich } \\
\text { hoher Zuwachs }\end{array}$ & $\begin{array}{l}\text { D: grosse Nach- } \\
\text { frage nach } \\
\text { Nadelrundholz }\end{array}$ & $\begin{array}{c}\text { E: grosse Nach- } \\
\text { frage nach } \\
\text { Energie- und } \\
\text { Chemieholz }\end{array}$ \\
\hline \multirow[t]{4}{*}{ Vorrat (m³/ha) } & $2007-2026$ & 350 & 359 & 341 & 333 & 341 \\
\hline & $2027-2056$ & 352 & 401 & 305 & 264 & 264 \\
\hline & 2057-2106 & 354 & 471 & 305 & 302 & 281 \\
\hline & $2007-2106$ & 353 & 428 & 312 & 297 & 288 \\
\hline \multirow{4}{*}{$\begin{array}{l}\text { Bruttozuwachs } \\
\left.\left(\mathrm{m}^{3} /[\text { hax }]\right]\right)\end{array}$} & $2007-2026$ & 8.85 & 8.94 & 8.77 & 8.73 & 8.81 \\
\hline & $2027-2056$ & 8.49 & 9.07 & 7.79 & 7.49 & 7.02 \\
\hline & $2057-2106$ & 8.99 & 10.08 & 8.70 & 9.66 & 9.08 \\
\hline & $2007-2106$ & 8.81 & 9.55 & 8.44 & 8.82 & 8.41 \\
\hline \multirow{4}{*}{$\begin{array}{l}\text { Abgang } \\
\left.\left(\mathrm{m}^{3} /[\text { hax }]\right]\right)\end{array}$} & $2007-2026$ & 9.22 & 8.42 & 10.05 & 10.73 & 10.49 \\
\hline & $2027-2056$ & 8.24 & 7.27 & 8.67 & 9.25 & 9.29 \\
\hline & $2057-2106$ & 8.76 & 8.01 & 8.36 & 8.19 & 7.88 \\
\hline & 2007-2106 & 8.70 & 7.87 & 8.79 & 9.02 & 8.82 \\
\hline
\end{tabular}

Tab 2 Für die Schweiz über den angegebenen Zeitraum gemittelte Werte zu Vorrat, Bruttozuwachs und Abgang (Summe von Nutzung und Mortalität) in Abhängigkeit des Bewirtschaftungsszenarios. Alle Angaben in Schaftholz.

2006). Aktuelle Ergebnisse aus der Inventur LFI4b (2009/2013) wurden als Grundlage für die Definition der Szenarien A und B berücksichtigt. Der Vorrat gemäss LFI4b diente zudem für alle Szenarien als Basis für die Waldbewirtschaftung während des ersten Simulationszeitschritts (2007-2016), die so eingestellt wurde, dass die beobachteten Vorräte von LFI4b erreicht wurden.

Wenn keine grossflächigen Störungsereignisse auftreten, beträgt die natürliche Mortalität im Schweizer Wald ungefähr 15\% der Summe aller Abgänge. Diese Mortalitätsrate wurde zwischen LFI1 und LFI2 (Brassel \& Brändli 1999) und zwischen LFI3 und LFI4b beobachtet. Für die Szenarien A, B und C wurde angenommen, dass die Mortalität weiterhin in diesem Bereich liegt, während für die Szenarien $\mathrm{D}$ und $\mathrm{E}$ aufgrund des starken Vorratsabbaus eine tiefere Mortalitätsrate (10\%) angenommen wurde (Tabelle 1). Die Mortalität zwischen LFI2 und LFI3 war durch den Sturm Lothar und seine Folgeschäden mit 21\% viel höher (Brändli et al 2010) und wurde nicht für die Simulationen verwendet.

Für die vorliegenden Szenarien wurde die Baumartenmischung in der Verjüngung an die Waldgesellschaften gemäss NaiS angepasst, weshalb für kolline bis untermontane Lagen keine reinen Nadelholzbestände simuliert wurden. Für das Szenario D wurde mit dem maximal zulässigen Nadelholzanteil verjüngt, während für die übrigen Szenarien mit den empfohlenen Werten (die etwas tiefer liegen) verjüngt wurde (Tabelle 1). Klimabedingte Veränderungen der Baumartenmischung wurden nicht berücksichtigt.

Für alle Szenarien wurden Sturmschäden simuliert, wobei sich Periodizität (15 Jahre) und Schadenausmass an Beobachtungen (Referenzwerte: Wintersturm 1967, Vivian 1990, Lothar 1999) orientieren (Pfister 1999). Ob während einer Dekade Sturmschä- den eintreffen, wo sich diese befinden und wie gross deren Ausmass (Anzahl betroffene Probeflächen) ist, wird in Massimo stochastisch bestimmt.

\section{Resultate}

Aus den Simulationen mit Massimo resultieren Ergebnisse für alle 14 Wirtschaftsregionen der Schweiz, wobei diese zusätzlich nach Nadelholz und Laubholz aufgegliedert werden können. Aus Gründen der Übersichtlichkeit erfolgt in diesem Beitrag eine zu Produktionsregionen aggregierte Darstellung der Ergebnisse.

\section{Waldentwicklung}

Die Vorgaben der Szenarien (Tabellen 1 und 2) konnten mehrheitlich erfüllt werden. Im gemeinsam zugänglichen Wald LFI2 und LFI3 bestand der Vorrat im Jahr 2016 (350 m³/ha) zu etwa zwei Dritteln aus Nadelhölzern. Im Basisszenario A blieben Vorrat (Jahr 2106: $355 \mathrm{~m}^{3} / \mathrm{ha}$ ) und Mischungsgrad gleich (Abbildung 1, Tabelle 2). Im Szenario B wurde aufgrund konstanter Nutzung gesamtschweizerisch ein starker Vorratsanstieg auf $510 \mathrm{~m}^{3} /$ ha simuliert. Regional zeigte sich jedoch ein stark variables Bild. Im Mittelland zum Beispiel fiel der Vorrat bis 2086 auf $240 \mathrm{~m}^{3} /$ ha und stellte damit in dieser Produktionsregion den tiefsten Endvorrat aller Szenarien dar. Auf die Vorratsabsenkung in den Szenarien C, D und E folgte eine Phase mit starker Nutzungsreduktion. Anschliessend stieg die Nutzung in diesen Szenarien wieder an und überstieg diejenige des Basisszenarios A. Gleichzeitig nahm der Bruttozuwachs gegen Ende des Simulationszeitraums deutlicher zu (Abbildung 1). Unter allen Szenarien ausser A stieg der Vorrat auf der Alpensüdseite weiter an. Die schweizweiten Endvorräte der Szenarien C 

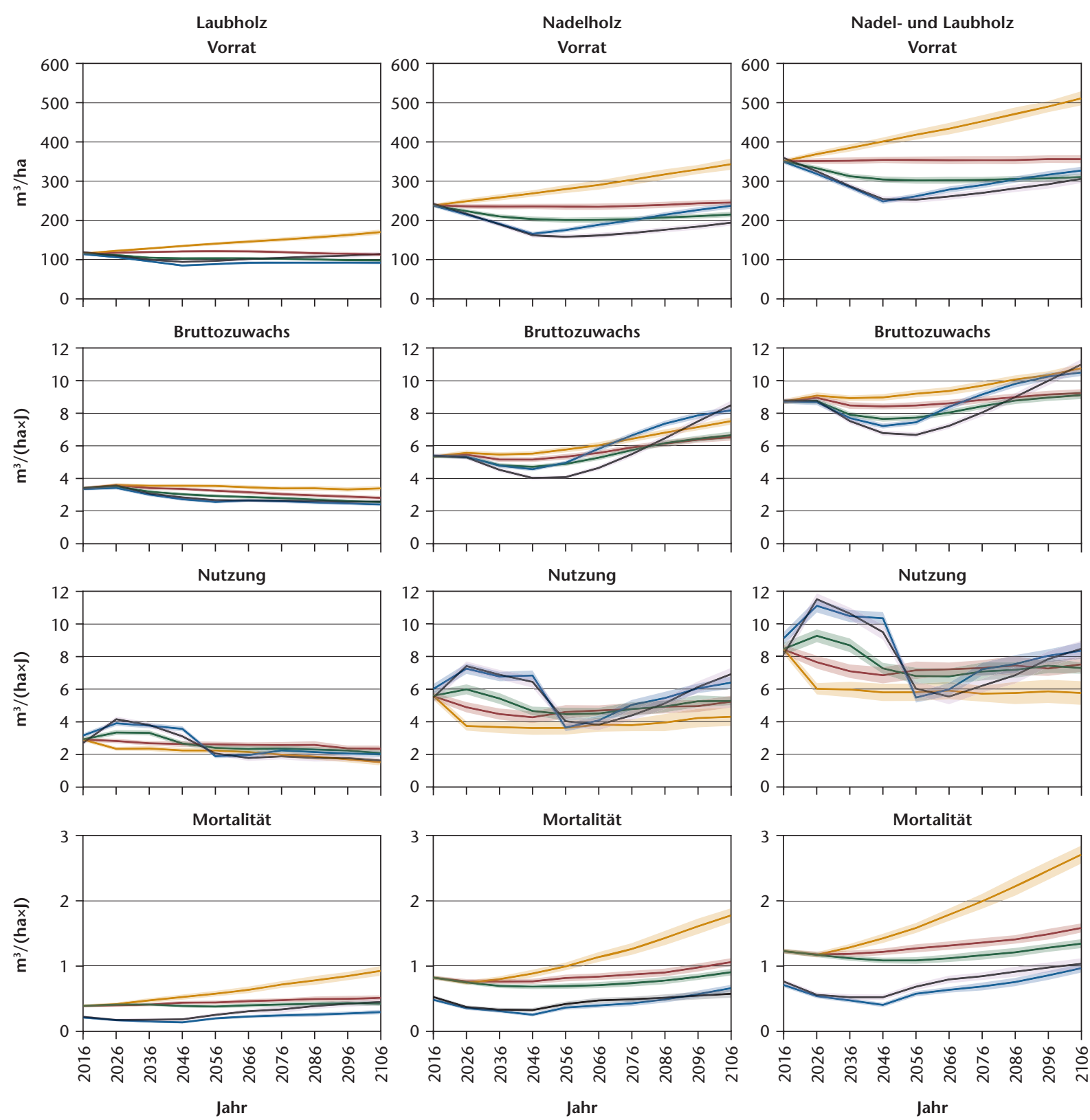

A: Basisszenario (konstanter Vorrat)

C: kontinuierlich hoher Zuwachs

D: grosse Nachfrage nach Nadelrundholz

E: grosse Nachfrage nach Energie- und Chemieholz

Abb 1 Entwicklung von Vorrat, Bruttozuwachs, Nutzung und Mortalität in der Schweiz bis 2106 für Laubholz, Nadelholz sowie für die Gesamtmenge in Abhängigkeit des Bewirtschaftungsszenarios. Alle Angaben in Schaftholz. Der Standardfehler ist mit transparenten Farbbereichen dargestellt. Die Ergebnisse zweier Szenarien unterscheiden sich statistisch signifikant für alle Zeitschritte, bei denen sich die Kurven bzw. der transparente Bereich nicht überschneiden.

( $\left.310 \mathrm{~m}^{3} / \mathrm{ha}\right)$ und D $\left(325 \mathrm{~m}^{3} / \mathrm{ha}\right)$ entsprachen den Szenariovorgaben. Bei Szenario E wich die Simulation nach 2046 von den Vorgaben ab: Der Vorrat stieg wegen Nutzungseinschränkungen in Reservaten, ungleichförmigen Wäldern und im Schutzwald bis Ende des Simulationszeitraums auf $304 \mathrm{~m}^{3} / \mathrm{ha}$ an.

Die Vorratsabsenkung bis 2046 in den Szenarien $\mathrm{C}\left(300 \mathrm{~m}^{3} / \mathrm{ha}\right), \mathrm{D}$ und $\mathrm{E}$ (je $250 \mathrm{~m}^{3} / \mathrm{ha}$ ) führte besonders beim Nadelholz zu starken Zuwachsverlusten über diesen Zeitraum (Abbildung 1). Der Bruttozuwachs nahm beim Laubholz in allen Szenarien ausser Szenario B über den gesamten Simulationszeitraum leicht ab. Ab 2046 stiegen der Nadelholzzuwachs und somit auch der gesamte Zuwachs stark an, wobei der Nadelholzuwachs im Szenario D reichte, um den bisherigen Zuwachsverlust zu kompensieren (Tabelle 2). Für die Szenarien $C$ und $\mathrm{E}$ resultierte dagegen ein mittlerer jährlicher Zuwachsverlust von $0.4 \mathrm{~m}^{3} / \mathrm{ha}$.

Der Abgang (Nutzung und Mortalität) war aufgrund der Vorratsabsenkung in den Szenarien C, D und E zwischen 2017 und 2046 stark erhöht und die Unterschiede zwischen den Szenarien waren in diesem Zeitraum am grössten (Abbildung 1). Die Vorratsabsenkung führte zudem zu leicht sinkender Mortalität. Bis Ende des Simulationszeitraums stieg die Mortalität in allen Szenarien an, besonders stark im Szenario B. Die deutlich tieferen Startwerte für 

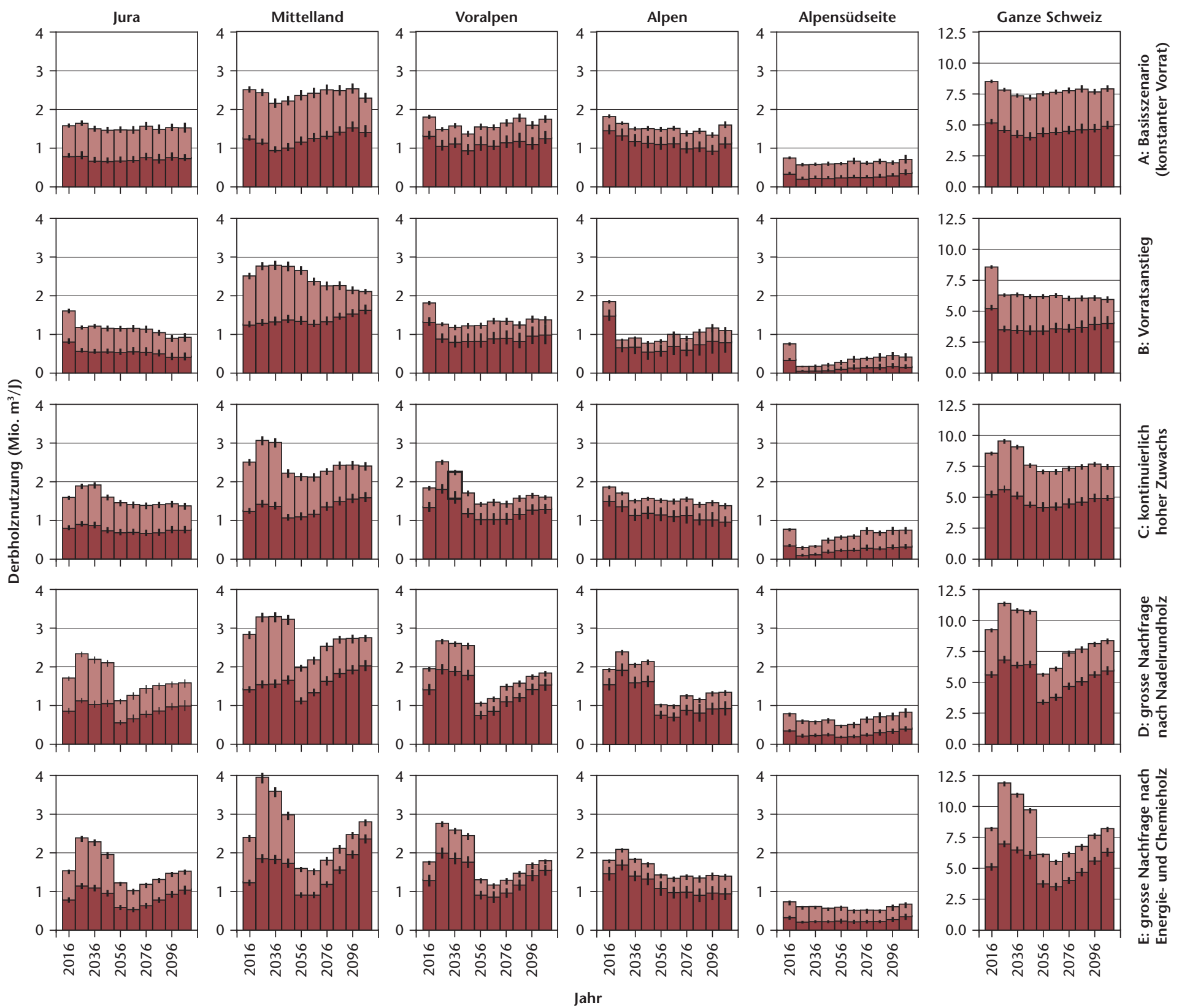

$\square$ Nadelholz $\square$ Laubholz

Abb 2 Entwicklung der jährlichen Derbholznutzung bis 2106 in den einzelnen Produktionsregionen und der Schweiz in Abhängigkeit des Bewirtschaftungsszenarios. Die schwarzen vertikalen Linien zeigen den Standardfehler.

die Mortalität bei den Szenarien D und E sind durch die Definition der Szenarien begründet. Der Abgang in den Szenarien C, D und E lag zwischen 2056 und 2106 etwas tiefer als in Szenario A, über den gesamten Simulationszeitraum hinweg war er - wegen der Vorratsabsenkung bis 2046 - aber deutlich höher (Tabelle 2).

\section{Derbholznutzung}

Im Basisszenario A blieb die Derbholznutzung in allen Produktionsregionen über den gesamten Simulationszeitraum einigermassen konstant bei etwa 7.7 Mio. $\mathrm{m}^{3} / \mathrm{J}$ (Abbildung 2). Dabei waren die absoluten (gesamtes Volumen) und relativen (pro Hektare) Nutzungsmengen im Mittelland am grössten und auf der Alpensüdseite am geringsten. Im Vergleich zum
Szenario A (768 Mio. $\mathrm{m}^{3}$ ) erhöhte sich die schweizweite Derbholnutzung mit den Szenarien C (kontinuierlich hoher Zuwachs: 783 Mio. $\mathrm{m}^{3},+2 \%$ ), D (grosse Nachfrage nach Nadelrundholz: 850 Mio. $\mathrm{m}^{3},+11 \%$ ) und E (grosse Nachfrage nach Energie- und Chemieholz: 808 Mio. $\mathrm{m}^{3},+5 \%$ ), während sie im Szenario B (631 Mio. $\mathrm{m}^{3}$ ) um 18\% geringer ausfiel (Tabelle 3).

Im Szenario B konnte die konstante Nutzung wie zwischen LFI3 und LFI4b im Mittelland nur bis 2046 erreicht werden, danach sank die Nutzung kontinuierlich ab. Auf der Alpensüdseite wurde über den gesamten Zeitraum eine steigende Nutzung simuliert, eine leichte Tendenz zu steigender Nutzung ergab sich auch in den Alpen. Im Jura und in den Voralpen blieb die Nutzung ab dem zweiten Simulationszeitschritt ziemlich konstant. 


\begin{tabular}{|c|c|c|c|c|c|c|}
\hline \multirow[t]{2}{*}{ Derbholznutzung } & \multirow[t]{2}{*}{ Zeitraum } & \multicolumn{5}{|c|}{ Szenario } \\
\hline & & $\begin{array}{l}\text { A: Basisszenario } \\
\text { (konstanter } \\
\text { Vorrat) }\end{array}$ & $\begin{array}{l}\text { B: Vorrats- } \\
\text { anstieg }\end{array}$ & $\begin{array}{l}\text { C: kontinuierlich } \\
\text { hoher Zuwachs }\end{array}$ & $\begin{array}{l}\text { D: grosse Nach- } \\
\text { frage nach } \\
\text { Nadelrundholz }\end{array}$ & $\begin{array}{l}\text { E: grosse Nach- } \\
\text { frage nach } \\
\text { Energie- und } \\
\text { Chemieholz }\end{array}$ \\
\hline \multirow{4}{*}{$\begin{array}{l}\text { jährliche Nutzung } \\
\left(\text { Mio. } \mathrm{m}^{3} / \mathrm{J} \text { ) }\right.\end{array}$} & $2007-2026$ & 8.1 & 7.4 & 9.0 & 10.3 & 10.0 \\
\hline & 2027-2056 & 7.3 & 6.1 & 7.9 & 9.0 & 8.9 \\
\hline & $2057-2106$ & 7.7 & 6.0 & 7.4 & 7.5 & 6.8 \\
\hline & $2007-2106$ & 7.7 & 6.3 & 7.8 & 8.5 & 8.1 \\
\hline \multirow{4}{*}{$\begin{array}{l}\text { Gesamtnutzung } \\
\left(\text { Mio. } \mathrm{m}^{3} \text { ) }\right.\end{array}$} & $2007-2026$ & 162 & 147 & 180 & 205 & 200 \\
\hline & $2027-2056$ & 219 & 184 & 236 & 271 & 267 \\
\hline & $2057-2106$ & 386 & 299 & 368 & 374 & 341 \\
\hline & $2007-2106$ & 768 & 631 & 783 & 850 & 808 \\
\hline
\end{tabular}

Tab 3 Für die Schweiz über den angegebenen Zeitraum gemittelte jährliche und aufsummierte Derbholznutzung in Abhängigkeit des Bewirtschaftungsszenarios.

\begin{tabular}{|c|c|c|c|c|c|c|}
\hline \multirow[t]{2}{*}{ Zeitraum } & \multirow{2}{*}{$\begin{array}{c}\text { Kostenklasse } \\
\left(\mathrm{CHF} / \mathrm{m}^{3}\right)\end{array}$} & \multicolumn{5}{|c|}{ Szenario } \\
\hline & & $\begin{array}{l}\text { A: Basisszenario } \\
\text { (konstanter } \\
\text { Vorrat) }\end{array}$ & $\begin{array}{l}\text { B: Vorrats- } \\
\text { anstieg }\end{array}$ & $\begin{array}{l}\text { C: kontinuierlich } \\
\text { hoher Zuwachs }\end{array}$ & $\begin{array}{l}\text { D: grosse Nach- } \\
\text { frage nach } \\
\text { Nadelrundholz }\end{array}$ & $\begin{array}{l}\text { E: grosse Nach- } \\
\text { frage nach } \\
\text { Energie- und } \\
\text { Chemieholz }\end{array}$ \\
\hline \multirow{3}{*}{$2007-2026$} & $<80$ & 3.9 & 3.8 & 4.5 & 5.1 & 5.2 \\
\hline & $80-150$ & 2.7 & 2.3 & 3.0 & 3.5 & 3.2 \\
\hline & $>150$ & 1.5 & 1.2 & 1.5 & 1.7 & 1.6 \\
\hline \multirow[t]{3}{*}{ 2027-2056 } & $<80$ & 3.4 & 3.4 & 3.8 & 4.3 & 4.1 \\
\hline & $80-150$ & 2.7 & 2.1 & 2.9 & 3.4 & 3.4 \\
\hline & $>150$ & 1.2 & 0.7 & 1.2 & 1.4 & 1.4 \\
\hline \multirow[t]{3}{*}{ 2057-2106 } & $<80$ & 3.4 & 2.4 & 2.7 & 2.3 & 1.6 \\
\hline & $80-150$ & 3.1 & 2.8 & 3.4 & 3.8 & 3.8 \\
\hline & $>150$ & 1.2 & 0.9 & 1.4 & 1.4 & 1.5 \\
\hline
\end{tabular}

Tab 4 Für die Schweiz über den angegebenen Zeitraum gemittelte jährliche Derbholznutzung (Mio. $\left.\mathrm{m}^{3} / J\right)$ je Kostenklasse in Abhängigkeit des Bewirtschaftungsszenarios.

Im Mittelland und in den Voralpen waren die Konsequenzen der Szenarien auf die Derbholznutzung besonders offensichtlich. Die Vorratsabsenkung bis 2046 gemäss den Szenarien C, D und E führt zu einer deutlich erhöhten Derbholznutzung in diesem Zeitraum (Abbildung 2). Im Szenario C reduzierte sich die Nutzung bereits ab 2026 kontinuierlich und pendelte sich ab 2056 bei etwa 7.4 Mio. $\mathrm{m}^{3} / \mathrm{J}$ ein. In den Szenarien D (Minimum 5.6 Mio. $\mathrm{m}^{3} / \mathrm{J}$ ) und E (Minimum 5.5 Mio. $\mathrm{m}^{3} / \mathrm{J}$ ) wurde die Nutzung zwischen 2047 und 2056 dagegen abrupt gesenkt. Bis Ende des Simulationszeitraums wurde die Nutzung parallel zum steigenden Vorrat und Zuwachs wieder erhöht. Daraus resultierte für das Szenario D von 2057 bis $2106 \mathrm{im}$ Durchschnitt eine Nutzung von $7.5 \mathrm{Mio.} \mathrm{m}^{3} / \mathrm{J}$ und für das Szenario E eine solche von 6.8 Mio. $\mathrm{m}^{3} / \mathrm{J}$.

\section{Holzerntekosten}

Je geringer die Holzerntekosten ausfallen, desto wahrscheinlicher ist es, dass ein Eingriff wirtschaftlich rentabel (Holzerlös + allfällige Beiträge decken die Kosten) ausgeführt werden kann. Unabhängig vom Szenario wurden im Jura und im Mittelland die geringsten Kosten simuliert (Abbildung 3), und Erntekosten von über $150 \mathrm{CHF} / \mathrm{m}^{3}$ kamen praktisch nicht vor. Ebenfalls szenariounabhängig stiegen im Mittelland die Erntekosten im Simulationszeitraum durchwegs an: So konnten bis 2046 rund drei Viertel der Holzernten für weniger als $80 \mathrm{CHF} / \mathrm{m}^{3}$ realisiert werden, gegen Ende des Simulationszeitraums waren es hingegen in Abhängigkeit des Szenarios nur noch 25 bis 55\%. Im Jura waren die Holzerntekosten und deren Entwicklung vergleichbar mit der Situation im Mittelland. In den Voralpen, Alpen und insbesondere auf der Alpensüdseite waren die Holzerntekosten deutlich höher. In den Voralpen konnten zu Beginn der Simulation 80\% der Holzernte für weniger als $150 \mathrm{CHF} / \mathrm{m}^{3}$ und etwa ein Drittel unter $80 \mathrm{CHF} / \mathrm{m}^{3}$ ausgeführt werden. Für die Szenarien A und B blieb dieses Verhältnis in etwa konstant, während die Vorratsabsenkung in den Szenarien C, D und E zu steigenden Holzerntekosten führte. Im Alpenraum waren die Erntekosten in allen Szenarien und über den gesamten Simulationszeitraum einigermassen konstant, wobei insgesamt etwa die Hälfte der Holzernte für unter $150 \mathrm{CHF} / \mathrm{m}^{3}$ und etwa $15 \%$ für weniger als $80 \mathrm{CHF} / \mathrm{m}^{3}$ realisiert werden konnten. Noch höhere Erntekosten wurden auf der Alpensüdseite berechnet, wo lediglich ein Viertel des Derbholzes unter $150 \mathrm{CHF} / \mathrm{m}^{3}$ genutzt werden konnte. Für die Schweiz als Ganzes war der Anteil 

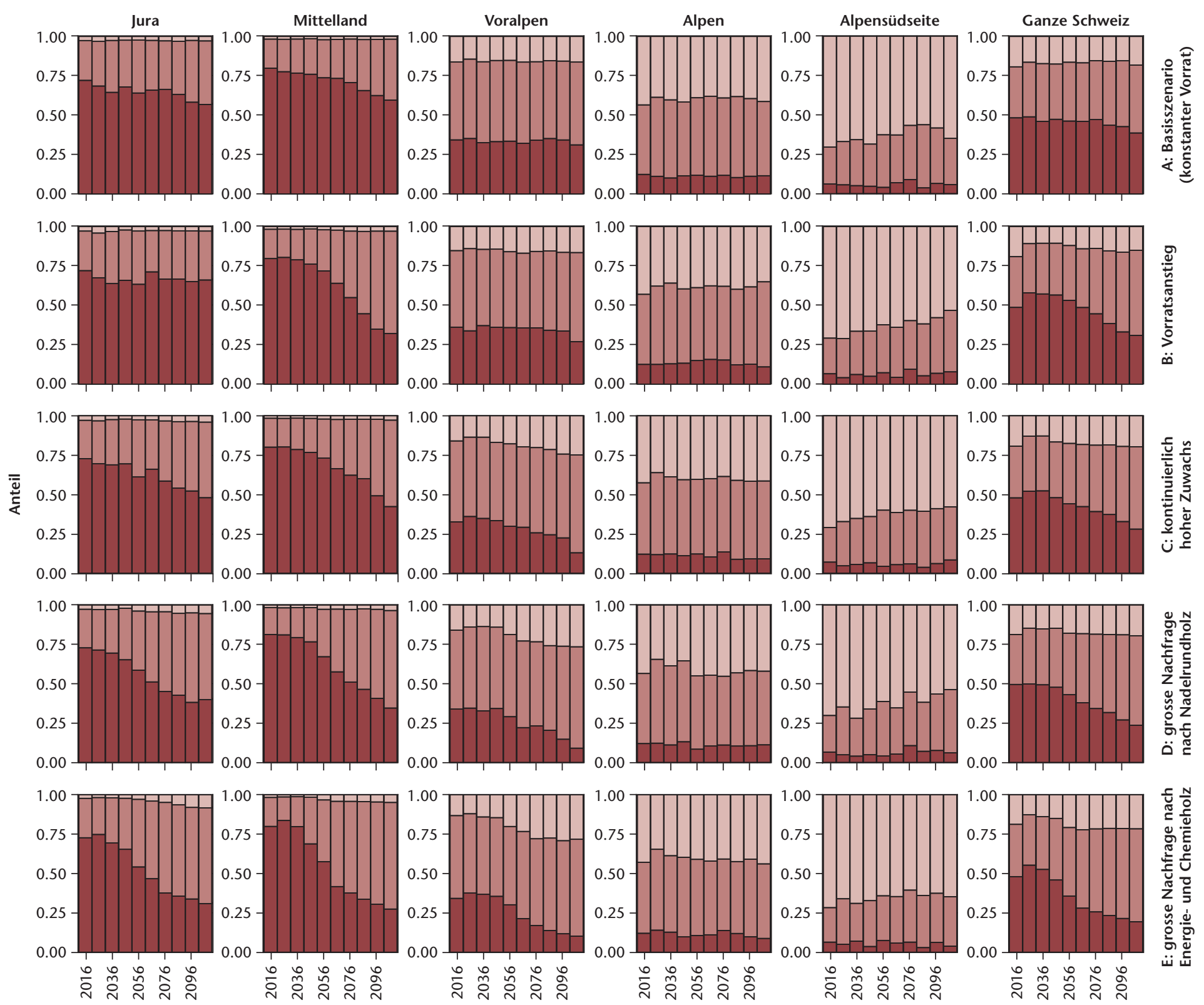

Jahr

Kostenklasse $\square<80 \mathrm{CHF} / \mathrm{m}^{3} \quad \square 80-150 \mathrm{CHF} / \mathrm{m}^{3} \quad \square>150 \mathrm{CHF} / \mathrm{m}^{3}$

Abb 3 Entwicklung der Anteile der Derbholznutzung je Kostenklasse bis 2106 in den einzelnen Produktionsregionen und für die Schweiz in Abhängigkeit des Bewirtschaftungsszenarios.

des in der Kostenklasse unter $80 \mathrm{CHF} / \mathrm{m}^{3}$ nutzbaren Derbholzes bei den Szenarien A (46\%) und B (47\%) am grössten. Die absolute Erntemenge in dieser Kostenklasse blieb im Szenario A ab 2026 konstant bei 3.4 Mio. $\mathrm{m}^{3} / \mathrm{J}$ und war insgesamt, d.h. über den Simulationszeitraum von 100 Jahren hinweg, am grössten (Tabelle 4), während die günstig verfügbaren Erntemengen in den übrigen Szenarien nach 2056 deutlich geringer ausfielen.

\section{Diskussion}

\section{Wie genau sind die Ergebnisse?}

Die Simulationsresultate sind mit Unsicherheiten behaftet, als Mass hierfür wurde der Standardfehler verwendet. Dieser setzt sich zusammen aus dem Stichprobenfehler sowie der Streuung, die sich durch die 20 Wiederholungen der Simulation eines Szenarios ergibt. Mit zunehmendem Simulationszeitraum (d.h. mit steigender Anzahl Zeitschritte) nimmt die Streuung zu. Nicht berücksichtigt wird die Unsicherheit der geschätzten Modellparameter, der Einflussgrössen und allfälliger ungenauer oder unkorrekter Modellannahmen. Auch diese Unsicherheiten akkumulieren sich über die Zeit. Die statistisch geschätzten Unsicherheiten sind somit zu optimistisch. Zudem ist es in der Praxis unmöglich, den Wald während 100 Jahren konsequent in gleicher Art und Weise zu bewirtschaften. Dennoch wurde dieser lange Zeitraum gewählt, denn nur so können die Auswirkungen heutiger Bewirtschaftungsentscheide auf das langsam reagierende System der Waldentwicklung langfristig abgeschätzt werden. 


\section{Welche wirtschaftlichen Auswirkungen zeigen die Szenarien?}

Im Basisszenario A wird der Vorrat in allen Wirtschaftsregionen konstant gehalten, indem die Summe aller Abgänge (Nutzung und Mortalität) jeweils dem Bruttozuwachs entspricht. Dabei kann jedes Jahr etwa die gleiche Holzmenge geerntet werden. Die Verfügbarkeit und die Erntekosten für Derbholz sind leicht abzuschätzen und keinen grossen Schwankungen ausgesetzt.

Beim Weiterführen der bisherigen Nutzung (Szenario B) ist die Verfügbarkeit von Derbholz im Prinzip langfristig gewährleistet, da nur ein Teil des schweizweiten Zuwachses genutzt wird. Dies gilt allerdings nicht für das Mittelland. Der in der Schweiz heute zu tiefe Flächenanteil an jungen Altersklassen (Brändli \& Cioldi 2015) sinkt ausserhalb des Mittellands weiter, während insbesondere in den Alpen und auf der Alpensüdseite der zu hohe Anteil an Altbeständen weiter steigt. Dadurch nimmt das Starkholz weiter zu, das sich gegenwärtig schlecht vermarkten lässt. Der starke Vorratsabbau im Mittelland dürfte hingegen dazu führen, dass Zuwachsverluste resultieren und weniger Baumholz produziert werden kann. Zudem bricht im Mittelland die Verfügbarkeit von Derbholz mit geringen Erntekosten ein. Insgesamt fällt die Holznutzung in diesem Szenario 18\% tiefer aus als im Basisszenario A bei gleichzeitigem Ansteigen der Holzerntekosten.

In den Szenarien C, D und E wird der Vorrat bis 2046 reduziert. Dazu wird die Nutzung erhöht. Im Szenario C (kontinuierlich hoher Zuwachs) erfolgt anschliessend eine schrittweise Nutzungsreduktion, die Nutzung pendelt sich danach auf dem Niveau des Basisszenarios A ein. Bei den Szenarien D und E wird die Nutzung hingegen stärker erhöht und danach auf einen Schlag reduziert. Diese schlagartige Nutzungsreduktion dürfte zu Versorgungsschwierigkeiten für die Holzverarbeiter führen. Nach 2056 wird die Nutzung laufend wieder gesteigert, ohne dass sich ein Gleichgewicht einstellt. Die Szenarien C, D und E führen zwar zu einer Vorratsabnahme, ermöglichen aber langfristig grössere Nutzungsmengen. Während des Vorratsabbaus bis 2046 sinkt der Anteil von günstig verfügbarem Holz, mit der Folge, dass wesentliche Nutzungsmengen bei Kosten von mehr als $80 \mathrm{CHF} / \mathrm{m}^{3}$ geerntet werden müssten, was heute aufgrund der tiefen Erlöse oft nicht wirtschaftlich umsetzbar ist. Dieser Trend setzt sich bis Ende des Simulationszeitraums fort.

\section{Können Risiken vermindert werden?}

Die Simulationen mit Massimo haben gezeigt, dass ein Weiterführen der bisherigen Bewirtschaftung (Szenario B) längerfristig mit Risiken verbunden ist. Die Bestände dürften dichter und gedrängter werden, mit negativen Folgen für deren Stabilität (Brändli \& Cioldi 2015). Im Szenario B dürfte somit das Risiko für störungsbedingte Waldschäden wie Sturm- oder Schneebruch und damit verbunden für Borkenkäferschäden steigen (Stadelmann et al 2014). Die Störungsresistenz und die Resilienz des Waldes dürften steigen, wenn die Vorräte in den Wäldern des Schweizer Alpenraums stabilisiert oder gesenkt würden. Im Jura und im Mittelland ist diesbezüglich keine weitere Vorratsreduktion notwendig. Vielmehr kann eine weitere Vorratsreduktion im Mittelland (insbesondere in der Wirtschaftsregion Mittelland Mitte) dazu führen, dass das Holzerntepotenzial der Schweiz abnimmt. Alternative Bewirtschaftungsszenarien könnten ein Ungleichgewicht der Durchmesserklassen im gleichförmigen Hochwald verhindern und dabei die Verfügbarkeit von Derbholz aus allen Sortimentsklassen steigern. Der dazu notwendige Vorratsabbau an Lagen mit hohen Erntekosten dürfte jedoch kaum durch die Erlöse aus dem Holzverkauf finanzierbar sein.

\section{Schlussfolgerungen}

Die Bewirtschaftungsszenarien zeigen einen breiten Handlungsspielraum auf, der dazu beitragen kann, geeignete regionale und nationale Strategien zur Vorrats- und Nutzungsplanung zu finden. Es gibt nicht das beste Szenario, das auf nationaler Ebene umgesetzt werden kann. Eine Stabilisierung des Vorrats (Szenario A) könnte die Resistenz des Waldes im Alpenraum verbessern, ist jedoch mit hohen Erntekosten je Kubikmeter verbunden. Das Fortführen des Szenarios B dürfte hingegen die nationale Holzverfügbarkeit in günstigen Kostenklassen und die Stabilität des Waldes im Alpenraum am stärksten einschränken. Aus Gründen der Versorgungssicherheit sind zudem Bewirtschaftungsszenarien mit schlagartiger, starker Nutzungsreduktion (Szenarien D und E) als ungünstig zu beurteilen. Das Festlegen von Mindest- und Maximalvorräten für den Wirtschaftswald jeder Wirtschaftsregion könnte zur langfristigen Sicherung der Holzerntepotenziale beitragen.

Eingereicht: 22. Oktober 2015, akzeptiert (mit Review): 29. Februar 2016

\section{Literatur}

BÖSCH B (2004) Das künftige Holzaufkommen in Baden-Württemberg. Freiburg i Br: Forstliche Versuchs- Forsch.anstalt Baden-Württemberg, FVA-Einblick 4: 10-13.

BRÄNDLI UB, CIOLDI F (2015) Altersaufbau und Bestandesstruktur. In: Rigling A, Schaffer HP, editors. Waldbericht 2015. Zustand und Nutzung des Schweizer Waldes. Bern: Bundesamt Umwelt. pp. 34-37.

BRÄNDLI UB, EDITOR (2010) Schweizerisches Landesforstinventar. Ergebnisse der dritten Erhebung 2004-2006. Birmensdorf: Eidgenöss Forsch.anstalt WSL. 312 p.

BRASSEL P, BRÄNDLI UB, EDITORS (1999) Schweizerisches Lan desforstinventar. Ergebnisse der Zweitaufnahme 1993-1995. Bern: Haupt. 442 p. 
CAMIN P, CIOLDI F, RÖÖSLI B (2015) Holzvorrat. In: Rigling A, Schaffer $\mathrm{H}$, editors. Waldbericht 2015. Zustand und Nutzung des Schweizer Waldes. Bern: Bundesamt Umwelt. pp. 32-33.

FISCHER C, CAMIN P (2015) Holznutzung und Zuwachs. In: Rigling $A$, Schaffer $H$, editors. Waldbericht 2015. Zustand und Nutzung des Schweizer Waldes. Bern: Bundesamt Umwelt. pp. $60-61$

FOEN (2015) Switzerland's greenhouse gas inventory 1990-2013: National inventory report including reporting elements under the Kyoto Protocol. Submission of 15 April 2015 under the UNFCCC and under the Kyoto Protocol. Bern: Federal Office Environment. $596 \mathrm{p}$.

FREHNER M, WASSER B, SCHWITTER R (2005) Nachhaltigkeit und Erfolgskontrolle im Schutzwald. Wegleitung für Pflegemassnahmen in Wäldern mit Schutzfunktion. Bern: Bundesamt Umwelt Wald Landschaft. $564 \mathrm{p}$.

HOFER P, ALTWEGG J, SCHOOP A, HÄSSIG J, RÜEGG R ET AL (2011) Holznutzungspotentiale im Schweizer Wald. Auswertung von Nutzungsszenarien und Waldwachstumsentwicklung. Bern: Bundesamt Umwelt, Umwelt-Wissen 1116. $80 \mathrm{p}$.

KAUFMANN E (2001) Prognosis and management scenarios. In: Brassel $P$, Lischke $H$, editors. Swiss national forest inventory: methods and models of the second assessment. Birmensdorf: Swiss Federal Research Institute WSL. pp. 197-206.

KAUFMANN E (2011) Nachhaltiges Holzproduktionspotenzial im Schweizer Wald. Schweiz Z Forstwes 162: 300-311. doi: 10.3188/szf.2011.0300

MONSERUD RA, STERBA H (1996) A basal area increment model for individual trees growing in even- and uneven-aged forest stands in Austria. For Ecol Manage 80: 57-80.

NABUURS GJ, SCHELHAAS MJ, PUSSINEN A (2000) Validation of the European forest information scenario model (EFISCEN) and a projection of Finnish forests. Silva Fenn 34: 167-179.

PFISTER C (1999) Wetternachhersage. 500 Jahre Klimavariationen und Naturkatastrophen (1496-1995): Bern: Haupt. 304 p.

\section{Potentiel de la récolte des bois dans la forêt suisse: simulation de scénarios d'exploitation}

Au cours de la dernière décennie, le volume de bois en Suisse a augmenté de 3\%, avec un accroissement notable dans I'Arc alpin, tandis que le volume par hectare a diminué sur le Plateau. Avec Massimo, modèle de développement de la forêt, nous avons simulé cinq scénarios d'exploitation sur une période de 100 ans. Dans le scénario de base «volume constant», il est possible de récolter chaque année 7.7 millions de $\mathrm{m}^{3}$ de bois fort en moyenne. Le potentiel de récolte est inférieur dans le scénario «accroissement du volume» $\left(6.3\right.$ millions de $\left.\mathrm{m}^{3}\right)$. Des potentiels de récolte supérieurs résultent des scénarios «croissance élevée continue» ( 7.8 millions de $\left.\mathrm{m}^{3}\right)$, «grande demande de bois de résineux» ( 8.5 millions de $\mathrm{m}^{3}$ ) et «grande demande de bois-énergie et de bois pour l'industrie chimique» ( $8.1 \mathrm{mil}$ lions de $\mathrm{m}^{3}$ ). Dans tous les scénarios, les coûts de la récolte par mètre cube augmentent avec le temps, cet effet étant le moins marqué dans le scénario de base. La poursuite de l'exploitation actuelle (scénario accroissement du volume) engendrera probablement, en raison de l'augmentation de la densité des peuplements et des volumes, un risque supérieur de perturbations (vent, bris de neige, scolytes), tandis que la surconsommation régionale sur le Plateau limitera la disponibilité de bois. Dans I'Arc alpin, une stabilisation des volumes (scénario volume constant) pourrait améliorer la résistance de la forêt, mais elle entraînerait des coûts de récolte élevés par mètre cube. Les scénarios d'exploitation visent à aider les décideurs à déterminer des stratégies adaptées pour la planification des volumes de bois et de l'exploitation, et à en évaluer les coûts.
STADELMANN G, BUGMANN H, WERMELINGER B, BIGLER C (2014) Spatial interactions between storm damage and subsequent infestations by the European spruce bark beetle. For Ecol Manage 318: 167-174.

STADELMANN G, TEMPERLI C, CONEDERA M, GÓMEZ A, BRANG P (2015) Möglichkeiten zur Holzmobilisierung im Tessiner Kastaniengürtel. Schweiz Z Forstwes 166: 291-298. doi: 10.3188/ szf.2015.0291

THEES O, KAUFMANN E, LEMM R, BÜRGI A (2013) Energieholzpotenziale im Schweizer Wald. Schweiz Z Forstwes 164: 351 364. doi: 10.3188/szf.2013.0351

TAVERNA R, GAUTSCHI M, HOFER P (2016) Das nachhaltig verfügbare Holznutzungspotenzial im Schweizer Wald. Schweiz Z Forstwes 167: 162-171. doi: 10.3188/szf.2016.0162

THÜRIG E, KAUFMANN E (2008) Waldbewirtschaftung zur Senkenerhöhung? Mögliche Konfliktfelder und Synergien. Schweiz Z Forstwes 159: 281-287. doi: 10.3188/szf.2008.0281

THÜRIG E, KAUFMANN E, FRISULLO R, BUGMANN H (2005) Evaluation of the growth function of an empirical forest scenario model. For Ecol Manage 204: 53-68.

WERNER F, TAVERNA R, HOFER P, THÜRIG E, KAUFMANN E (2010) National and global greenhouse gas dynamics of different forest management and wood use scenarios: a model-based assessment. Environ Sci Policy 13: 72-85.

\section{Quellen}

Abegg M, Brändli UB, Cioldi F, Fischer C, Herold-Bonardi A et al (2014) Viertes Schweizerisches Landesforstinventar - Ergebnistabellen und Karten im Internet zum LFI 2009-2013 (LFI4b). Birmensdorf: Eidgenöss Forsch.anstalt WSL. www.Ifi.ch/resultate (29.2.2016)

\section{Timber-harvesting potential of Swiss forests: simulation of management scenarios}

Growing stock in Swiss forests has, during the past 10 years, increased by $3 \%$ overall, with large regional variation. The increase in Alpine regions has been considerable, while growing stock has decreased on the Plateau. We simulated 5 different 100-year management scenarios with the forest development model Massimo. In the base scenario "constant growing stock", 7.7 million $\mathrm{m}^{3}$ of merchantable timber could be harvesting annually. In the scenario "increasing growing stock", the harvesting potential was lower (6.3 million $\left.\mathrm{m}^{3}\right)$, but it was higher in the scenarios "continually high increment" (7.8 million $\mathrm{m}^{3}$ ), "high demand for coniferous timber" (8.5 million $\mathrm{m}^{3}$ ) and "high demand for wood fuel or chemical timber" (8.1 million $\mathrm{m}^{3}$ ). With time, timber-harvesting costs increase for all scenarios, with the least increase for the base scenario. If the prevailing management system continues (scenario "increasing growing stock"), stands will become denser and growing stock increase, with the risk of more disturbances, e.g. wind, snow-breakage, or bark beetles. In the Plateau, however, the regional overuse of recent decades could limit the overall timber-harvesting potential. In Alpine regions, stabilizing growing stock (scenario "constant growing stock") could improve the resistance of forests, but the harvesting costs per cubic meter might be higher. These management scenarios provide decision makers with indications of how particular forest management strategies could affect the development of growing stock, harvesting amounts and costs. 